\title{
LA DECLARACIÓN DEL RIESGO EN EL SEGURO DE VIDA, DEBER DE INFORMAR Y DE INFORMARSE*
}

\section{INSURED PRECONTRACTUAL DUTY OF THE LIFE INSURANCE}

\section{ROBERTO RÍOS OSSA*}

Fecha de recepción: 13 de julio de 2017

Fecha de aceptación: 15 de agosto de 2017

\section{Para Citar este articulo/To cite this article}

Ríos Ossa, Roberto, La declaración del riesgo en el seguro de vida, deber de informar y de informarse, 47 Rev.Ibero-Latinoam.Seguros, 107-118 (2017). https://doi.org/10.11144/Javeriana.ris47.drsv

doi:10.11144/Javeriana.ris47.drsv

* Artículo de reflexión, desarrollado a partir de la conferencia dictada por el autor en el marco ponencia de la sección nacional de Chilena en el XIV Congreso del Comité Iboerolatinoamericano de AIDA CILA. Santa Cruz de la Sierra - Bolivia. 2 a 5 de mayo de 2017.

** Abogado, Doctor en derecho privado por la Pontificia Universidad Católica de Chile. Profesor de derecho comercial y director del Programa de Seguros de la Pontificia Universidad Católica de Chile. Miembro de Aida-Chile. rrioso@uc.cl. 


\section{RESUMEN}

El deber de declaración del riesgo asegurado en el ordenamiento jurídico chileno transitó de un sistema de declaración espontáneo a uno guiado por cuestionario, según las nuevas exigencias legales introducidas por la ley 20.667 a la regulación del contrato de seguro, que recoge, además, el principio favor contractus.

Palabras clave: declaración del riesgo; cuestionario; seguros de vida. 


\section{ABSTRACT}

The Chilean law on insured precontractual duty changed to the questionnaire system. Additionally, the new regulation rescued the principle of favor contractus.

Keywords: declaration of risk; questionnaire; life insurance.

\section{SUMARIO}

INTRODUCCIÓN. 1. EL DEBER PRECONTRACTUAL DE DECLARACIÓN DEL RIESGO ASEGURABLE. 1.1. Forma del deber precontractual de declaración del riesgo. 1.2. Efectos derivados del incumplimiento al deber precontractual de declaración del riesgo. 1.2.1. La declaración inexacta, reticente o errónea. Unidad de supuesto básico del artículo 525 del C.com. 1.2.1.1. La inexactitud, la reticencia y el error. 1.2.1.2. Efectos de la Declaración inexacta, reticente o errónea. 1.2.2. Declaración de mala fe o sustancialmente falsa. Unidad de supuesto básico del artículo 539 del C.com. 2. EPÍLOGO. BIBLIOGRAFÍA 


\section{INTRODUCCIÓN}

El año 2013 la regulación del contrato de seguro en Chile fue sustituida en su integridad al incorporarse un nuevo contenido normativo en el título VIII del libro II del Código de Comercio chileno (en adelante C.com.). Para nosotros, las innovaciones de mayor relevancia -en especial por su transversalidad- están representadas en la imperatividad de las normas que regulan el contrato de seguro ${ }^{1}$, por un lado, y por el otro, en especiales deberes precontractuales de información ${ }^{2}$. Ambos instrumentos poseen un objetivo tutelar común a favor del asegurado ${ }^{3}$.

1 Sobre la imperatividad en el derecho chileno ver por todos Ríos, Roberto (2015): “Artículo 542, Carácter imperativo de las normas, en Ríos Roberto (director), El Contrato de seguro, Comentarios al Título VIII Libro II del Código de Comercio (Santiago, Thomson Reuters), p. 461.

2 La relevancia de la fase in contrahendo del negocio jurídico, la muestra por primera vez el notable Ihering, en su obra "Culpa in contrahendo oder Schadensersatz bei nichtigen oder nicht zur Perfection gelangten Verträgen”. La preocupación de Ihering por la fase de formación del contrato, surge de la teoría de error, los problemas que tal vicio del consentimiento involucra, y la imposibilidad de encontrar, según este autor, una respuesta satisfactoria. Luego, nos encontraremos con la obra de Leonhard, Die Haftung des Verkäufers für sein Verschulden beim Vertragsschlnsse. Este autor planteó considerar la culpa de uno de los contratantes antes y durante la celebración del contrato (vor und bei dem Vertragsschlusse), lo que amplía el espectro de análisis al formulado por Ihering, que se centra en el momento mismo de la conclusión del acuerdo. Leonhard, desarrollaró su tesis sobre la base del silencio culposo en las deficiencias o defectos de la mercancía en la compraventa, dado que, en su opinión, a través de él se puede seguir el desarrollo del principio central utilizado en viejos tiempos. En este autor encontraremos el tratamiento de dos temas centrales que advierten un análisis de la etapa precontractual y la existencia de deberes durante esta fase de formación del contrato. El primero de ellos, aparece a propósito de la advertencia de Cicerón sobre las simples recomendaciones y sobre el carácter obligacional de la promesa informal. Analiza Leonhard la distinción entre los dictum con caracteres vinculantes y aquellos que no tiene tal carácter por tratarse de simples recomendaciones. Vemos en este pasaje de la obra de Leonhard el reconocimiento a declaraciones que no vinculan, en consecuencia se refiere a lo que la moderna doctrina denomina como tratos previos. El declarante oculta dolosa o culposamente datos relevantes, señala este autor. En nuestra opinión, el análisis central de la tesis de Leonhard está en el cuestionamiento sobre la obligatoriedad (verbindlichkeit) que puede existir antes de la celebración del contrato. Se trata de una obligatoriedad relacionada con el cumplimiento de determinados deberes (Pflichten) del vendedor, destinados a evitar la conducta dolosa o culposa en la ocultación de vicios de la cosa (verschweigen von mängeln.). La conclusión de Leonhard es la siguiente: la justificación de los deberes (Pflichten) del vendedor está en su conducta negligente, ello es un principio jurídico de aplicación general, no sólo de aplicación exclusiva para el contrato de compraventa. En consecuencia, todo contratante está obligado al cumplimiento de determinados deberes de informar antes de la conclusión del contrato, debiendo responder de los daños que cause en el caso de incumplimiento. Siguiendo la línea de desarrollo de la doctrina alemana, encontraremos la siguiente secuencia. Primero la tesis de Siber, quien al referirse a la responsabilidad en la formación del contrato derivada del incumplimiento de deberes precontractuales de información, lo hace con independencia de la celebración o no de un contrato válido. Luego, Stoll introducirá adecuadamente la idea de relación jurídica preparatoria de la cual surgen los deberes precontractuales. Véanse Asua, Clara (1989): Culpa in contrahendo (San Sebastián, Universidad de País Vasco) pp. 55 y ss.; Ríos, Roberto (2014): El deber precontractual de declaración del riesgo en el seguro de daños (Santiago, Thomson-Reuters), pp. 14-15.

3 Véanse Contreras, Osvaldo (2014): Derecho de seguros (Santiago, Thomson Reuters), pp. 13-21; Hoyl, Rodrigo y Ruiz-Tagle, Carlos (2014): El contrato de seguro, análisis de las coberturas de vida 
El legislador chileno recogiendo las exigencias de la buena fe presente en el iter de formación del contrato, explicitó estos deberes de información, e incorporó nuevas normas sobre la forma, contenido y efectos derivados de su incumplimiento.

Nos abocaremos en esta oportunidad a analizar el deber precontractual de declaración del riesgo asegurado en sus diversas problemáticas. En primer lugar, daremos cuenta de los cambios en cuanto a la forma y contenido de esta carga o deber de información, (ii) en segundo lugar, abordaremos los efectos derivados de su incumplimiento, para (iii) finalmente, exponer nuestras conclusiones.

\section{EL DEBER PRECONTRACTUAL DE DECLARACIÓN DEL RIESGO ASEGURABLE}

El deber precontractual de información que recae en el contratante del seguro - deber o carga que tiene por finalidad suministrar de información al asegurador sobre el contorno del riesgo asegurable ${ }^{4}$ - se encuentra regulado en los artículos $524 \mathrm{~N}^{\mathrm{o}} 1,525$ y 539 del Código de comercio. Confluyen, además, los artículos 590, 591 y 592 para los casos de seguros de personas, normas legales que establecen reglas especiales en materia de preexistencias, informes médicos e indisputabilidad.

A continuación analizaremos los artículos 524 número 1, 525, 590 y 591, todos del C.com., que se refieren a la forma de materialización o cumplimiento de este especial deber de información en el caso de los denominados seguros de personas.

\subsection{Forma del deber precontractual de declaración del riesgo}

En cuanto a la forma del deber precontractual de declaración del riesgo, nuestro legislador recoge la práctica del sistema cuestionario en

e incendio en la nueva ley 20.667 (Santiago, Thomson-Reuters), pp. 1 y ss. La exposición de motivos de la ley 20.667, refiere claramente la calidad de consumidor del asegurado que contrata en términos de adhesión y bajo un sistema de condiciones generales de la contratación. Ver exposición de motivos en la tramitación de la ley 20.667, pp. 469-470.

4 Ríos, Roberto (2014): El deber precontractual de declaración del riesgo en el seguro de daños (Santiago, Thomson-Reuters), pp. 53 y ss. 
los artículos 524 número $1^{\circ} \mathrm{y}$ en el artículo 525, ambos del C.com., abandonando el sistema de declaración espontáneo ${ }^{5}$ que dejaba, por lo general, abierta la posibilidad de invocar de parte del asegurador un incumplimiento al deber precontractual de declaración del riesgo, cuando a juicio de este el contratante del seguro infringiendo las exigencias de la buena fe no entregó información sobre el riesgo que conocía o debía conocer y era relevante.

Frente a lo que hemos señalado supra, surge la interrogante sobre el rol de buena fe in contrahendo -o máxima buena fe como ha referido la doctrina tradicional ${ }^{6}$ - en un sistema que hemos calificado como cerrado, por un lado. Y por el otro, la interrogante apunta a la precisar la justificación del sistema.

En cuanto a la buena fe, el deber de comportamiento leal del contratante del seguro está representado en el responder de modo preciso y completo lo que se pregunta, sin más ${ }^{7}$. Por su parte, el sistema cuestionario cerrado se justifica en la relación asimétrica que existe entre el contratante del seguro y el asegurador. Este último no sólo es un experto en riesgos -especialmente en su evaluación-sino que posee una serie de instrumentos que le permiten obtener información, incluso anticiparse al contacto negocial inicial con el contratante. Ello impone al asegura-

5 En cuanto al sistema de declaración espontáneo, señaló Vivante que: Il codice impone all'assicurato due obblighi: $1^{\circ}$ di dire esattamente tutto quello che dice; $2^{\circ}$ di dire tutto quello che sa. Vivante, Cesare (1922): Del contratto di assicurazione (Torino, Unione Tipografico, Editrice-Torinese), p. 176.

6 Ver por todos Garrigues, Joaquín (1982): Contrato de seguro terrestre (Madrid, sin editorial), p. 46.

7 Se trata en este contexto y como lo ha calificado la doctrina de un deber de respuesta. Véanse Sánchez Calero, Fernando et al (2110): Artículo 10, Deber de declaración del riesgo, en Sánchez Calero (director) Ley de contrato de seguro, comentarios a la ley 50/1980 de 08 de octubre y sus modificaciones (Madrid, Thomson-Reuters), p. 282; Ríos, Roberto (2014): El deber precontractual de declaración del riesgo en el seguro de daños (Santiago, Thomson-Reuters), p. 48; Hoyl, Rodrigo y Ruiz-Tagle, Carlos (2014): El contrato de seguro, análisis de las coberturas de vida e incendio en la nueva ley 20.667 (Santiago, Thomson-Reuters), p. 148; Benito, Félix et al (2015): El contrato de seguro y las tecnologías aplicadas a la medicina y la salud, en IV Congreso de Nuevas Tecnologías, La influencia de internet, genética y nanotecnología en la medicina y en el seguro (Bogotá, Universidad Externado de Colombia), p. 99. En la tramitación parlamentaria de la ley 20.667, el ejecutivo presentó una indicación al artículo 525 sobre declaración del estado del riesgo que claramente sigue el texto del artículo 10 de la Ley de contrato de seguros española. El texto de la indicación es el siguiente: "Al celebrar el contrato de seguro, el contratante debe declarar al asegurador, de acuerdo con el cuestionario que éste disponga, las circunstancias por él conocidas que puedan influir en la valoración del riesgo. Quedará exonerado de tal deber si el asegurador no le somete al cuestionario o cuando, aún sometiéndolo, se trate de circunstancias que puedan influir en la valoración del riesgo y que no estén comprendidas en forma específica en dicho cuestionario". Ver historia de la ley 20.667 p. 46. 
dor como contrapartida al deber de declaración del riesgo una carga de autoinformarse, provocándose de este modo un cambio de paradigma en materia de información sobre el riesgo asegurable. En este sentido, la denominada máxima buena fe in contrahendo se impone recíprocamente a las partes, al asegurador como deber de elaborar un cuestionario preciso y claro ${ }^{8}$, y al contratante del seguro como un deber responder el cuestionario o formulario de preguntas proporcionado por el primero.

Para el caso concreto de los seguros de personas, debemos considerar que el factor subjetivo que integra el riesgo asegurado exige mayor precisión en la elaboración del cuestionario. El asegurador requiere conocer no sólo el estado de salud del asegurado, sino su conductas de vida asociadas al riesgo asegurable. Por otra parte, es de uso común en los sistemas de aseguramiento de vida o de riesgos asociados a la vida, salud o integridad física de las personas, complementar las respuestas del cuestionario con exámenes médicos, cuyos resultados se integran como una respuesta mas de cuestionario. Estos exámenes deben ajustarse a las exigencia legales, según lo prescribe el artículo 590 del C.com ${ }^{9}$, y sus resultados se integran como una respuesta más del deber de declaración.

Determinada la forma y contenido del deber precontractual de información del riesgo, es posible precisar un estado de incumplimiento y sus efectos. A ello no abocaremos a continuación.

\subsection{Efectos derivados del incumplimiento al deber precontractual de declaración del riesgo}

En cuanto de los efectos derivados del incumplimiento del deber de informar, nuestro legislador en los arts. 525 y 539 del C.com. recoge lo que hemos calificado como un nuevo régimen de vicios del consentimiento. La nulidad queda relegada sólo para declaraciones de mala fe. Luego y para aquellos casos en los que la declaración no contiene el elemento de la culpa o inexcusabilidad nuestro legislador opta por la conservación

8 En este sentido ver Ríos, Roberto (2014): El deber precontractual de declaración del riesgo en el seguro de daños (Santiago, Thomson-Reuters), pp. 38 y ss.

9 Hoyl, Rodrigo y Ruiz-Tagle, Carlos (2014): El contrato de seguro, análisis de las coberturas de vida e incendio en la nueva ley 20.667 (Santiago, Thomson-Reuters), p. 156. 
del contrato ${ }^{10}$, en caso contrario subiste un derecho de desistimiento a favor del asegurador.

De los artículos 525 y 539 podemos extraer dos tipos de incumplimiento al deber precontractual de declaración del riesgo. De citado artículo 525 la declaración errónea reticente a inexacta, y del artículo 539 la declaración de mala fe o sustancialmente falsa. Veamos cada una de ellas, y sus efectos. Este tipo de declaraciones defectuosas las calificamos como una unidad de supuesto básico que permiten abrir el sistema de remedios. Luego tendremos que identificar un supuesto complementario o factor cronológico -si ha ocurrido el siniestro o no- por un lado, y por el otro, un factor supuesto complementario de imputación o culpa que nuestro legislador ha enunciado con el vocablo inexcusables ${ }^{11}$. Con ello podremos preciar el efecto que provoca el incumplimiento al deber precontractual de declaración del riesgo.

\subsubsection{La declaración inexacta, reticente o errónea. Unidad de supuesto básico del artículo 525 del C.com}

\subsubsection{La inexactitud, la reticencia y el error}

De la lectura del artículo 525 del Código de Comercio podríamos sostener que el contratante del asegurado puede declarar de modo inexacto, reticente o erróneo, incumpliendo de esta manera con el deber impuesto en el artículo 524 número 1 del C.com.

Para nosotros, la declaración errónea es aquella que contiene datos que no corresponden a la realidad. Del mismo modo, la declaración inexacta

10 Donati ya advirtió sobre el reconocimiento del principio de conservación del contrato en su análisis de los artículos 1892 y 1893 del Codice Civile. Donati, Antigono (1954): Trattato del Diritto delle Assicurazionni Private (Milano, Guiffré), p. 300.

11 Nuestra formulación recoge la propuesta de Morales Moreno en materia de remedios derivados del incumplimiento contractual, que proponemos reconducir a la fase de formación del contrato. Morales Moreno, Antonio et al (2011): ¿Es posible construir un sistema precontractual de remedios? Reflexiones sobre la Propuesta de Modernización del Derecho de Obligaciones y Contratos en el marco del Derecho europeo, en Dohrmann, Albiez K. J. (director), Derecho privado europeo y modernización del Derecho contractual en España (Barcelona, Atelier), p. 409; Basozabal Arrue, Xabier et al (2012): Los deberes precontractuales de información después del DCFR, la Directiva 2011/83 y la Propuesta CÉSL, en Cámara, Sergio (Director) La revisión de las normas europeas y nacionales de protección de los consumidores más allá de la Directiva sobre derechos de los consumidores y del instrumento opcional sobre un Derecho europeo de la compraventa de octubre de 2011, (Madrid, Thomson Reuters), p. 190. 
obedece a un suministro de información que nos corresponde a la verdad ${ }^{12}$. Ambos supuestos, error e inexactitud se funden en u solo, apartándose del régimen común del error vicio del consentimiento que en nuestro derecho nos exige distinguir entre error esencial, sustancial y accidentes para los efectos de determinar si procede la nulidad y que tipo, absoluta o relativa.

En cuanto a la reticencia, podemos ver nuevamente un régimen especial apartado del sistema general de vicios del consentimiento. La declaración reticente del artículo 525 del Código de Comercio no queda, exclusivamente, vinculada al dolo. Se trata en este supuesto de una simple falta de entrega de información que fue requerida, no obstante el declarante omitió todo o en parte de preguntado.

\subsubsection{Efectos de la Declaración inexacta, reticente o errónea}

Verificada la ocurrencia de la unidad de supuesto básico representada por la declaración errónea, reticente o inexacta -que constituye el incumplimiento al deber de declaración del riesgo en su base in contrahendodebemos precisar si la inobservancia se verificó habiendo ocurrido el siniestro o riesgo asegurado o no -se trata del supuesto complementario o factor cronológico-, por un lado, y por otro lado, verificar si el contratante o tomador del seguro actuó de modo inexcusable ${ }^{13}$ o no -se trata del supuesto complementario o factor subjetivo-.

En el sentido que hemos señalado, si se verifica un supuesto de no ocurrencia del siniestro y conducta inexcusable, el asegurador podrá rescindir el contrato. Por rescisión entendemos una facultad de desistimiento que opera de pleno derecho ${ }^{14}$. Para aquellos casos en los que la

12 Ríos, Roberto (2014): El deber precontractual de declaración del riesgo en el seguro de daños (Santiago, Thomson-Reuters), p. 106.

13 Sobre el sentido del vocablo inexcusables del artículo 525 del Código de Comercio Ríos, Roberto (2014): El deber precontractual de declaración del riesgo en el seguro de daños (Santiago, ThomsonReuters) pp. 112 y ss.

14 El vocablo rescisión en nuestro derecho no es unívoco de nulidad relativa. Ver por todos Alcalde, Jaime et al (2009): La rescisión en el Código Civil chileno, en Estudios de Derecho Civil V (Santiago, Lexis Nexis) p. 77. En sentido contrario a propósito del artículo 525 del Código de Comercio véanse Arellano, Sergio (2013): La ley de Seguros (Santiago, Thomson Reuters) p. 70; Hoyl, Rodrigo y RuizTagle, Carlos (2014): El contrato de seguro, análisis de las coberturas de vida e incendio en la nueva ley 20.667 (Santiago, Thomson-Reuters), p 80. 
conducta del contratante o tomador del seguro no es inexcusable, opera la principio favor contractus, pudiendo en este caso ser modificadas las condiciones contractuales mediante un aumento de la prima o cambio en las condiciones de cobertura, como prescribe el citado artículo 525 del C.com.

Para los casos en los que se ha verificado el siniestro, el citado artículo 525 dispone la rescisión o desistimiento del contrato de parte del asegurador en el caso que concurra la inexcusabilidad, y en ausencia de ella, el asegurado o contratante tendrá derecho a la indemnización aunque reducida.

Es ineludible señalar, que la indisputabilidad del artículo 591 del C.com. en el caso de los seguros de personas, constituye un límite a los efectos del citado artículo $525^{15}$. Estos no operan, quedan atajados por los efectos del citado artículo 591.

\subsubsection{Declaración de mala fe o sustancialmente falsa. Unidad de supuesto básico del articulo 539 del C.com.}

El artículo 539 del C.com. contempla bajo la rúbrica "Otras causales de ineficacia", la declaración a sabiendas sustancialmente falsa. A nuestro juicio, esta norma considera tanto conducta dolosas como de mala fe, aspecto que estimados relevante destacar, ya que no toda mala fe involucra dolo; por el contrario, todo dolo es mala $\mathrm{fe}^{16}$.

\section{EPÍLOGO}

Nuestra tesis apunta en una doble perspectiva:

1) primero, la consagración de un sistema de declaración cuestionario, en el que la buena fe impone deberes correlativos a las partes; para

15 En este sentido en el derecho español ver Benito, Félix et al (2015): El contrato de seguro y las tecnologías aplicadas a la medicina y la salud, en IV Congreso de Nuevas Tecnologías, La influencia de internet, genética y nanotecnología en la medicina y en el seguro (Bogotá, Universidad Externado de Colombia), p. 102.

16 Véase Ríos, Roberto (2014): El deber precontractual de declaración del riesgo en el seguro de daños (Santiago, Thomson-Reuters) p. 119. 
el contratante del seguro, el deber de responder claramente las preguntas formuladas sobre el riesgo y para el asegurador, el deber de elaborar un cuestionario completo y claro.

2) En segundo lugar, apuntamos a la superación del sistema tradicional de vicios del consentimiento en materia de deberes de información precontractual en el seguro, al quedar relegada la nulidad a supuestos mínimos y favorecer la mantención del contrato, que no es otra cosa que buscar en el ideario de Ihering, el interés como motor de las relaciones jurídicas y por cierto su satisfacción.

\section{BIBLIOGRAFÍA}

Alcalde, Jaime et al ( 2009): La rescisión en el Código Civil chileno, en Estudios de Derecho Civil V (Santiago, Lexis Nexis).

Arellano, Sergio (2013): La ley de Seguros (Santiago, Thomson Reuters).

Asua, Clara (1989): Culpa in contrahendo (San Sebastián, Universidad de País Vasco)

Basozabal Arrue, Xabier et al (2012): Los deberes precontractuales de información después del DCFR, la Directiva 2011/83 y la Propuesta CÉSL, en Cámara, Sergio (Director) La revisión de las normas europeas y nacionales de protección de los consumidores más allá de la Directiva sobre derechos de los consumidores $y$ del instrumento opcional sobre un Derecho europeo de la compraventa de octubre de 2011, (Madrid, Thomson Reuters).

Benito, Félix et al (2015): El contrato de seguro y las tecnologías aplicadas a la medicina y la salud, en IV Congreso de Nuevas Tecnologías, La influencia de internet, genética y nanotecnología en la medicina y en el seguro (Bogotá, Universidad Externado de Colombia).

Contreras, Osvaldo (2014): Derecho de seguros (Santiago, Thomson Reuters).

Donati, Antigono (1954): Trattato del Diritto delle Assicurazionni Private (Milano, Guiffré).

Hoyl, Rodrigo y Ruiz-Tagle, Carlos (2014): El contrato de seguro, análisis de las coberturas de vida e incendio en la nueva ley 20.667 (Santiago, Thomson-Reuters).

Garrigues, Joaquín (1982): Contrato de seguro terrestre (Madrid, sin editorial)

Morales Moreno, Antonio et al (2011): ¿Es posible construir un sistema precontractual de remedios? Reflexiones sobre la Propuesta de Modernización del Derecho 
de Obligaciones y Contratos en el marco del Derecho europeo, en Dohrmann, Albiez K. J. (director), Derecho privado europeo y modernización del Derecho contractual en España (Barcelona, Atelier).

Ríos, Roberto (2014): El deber precontractual de declaración del riesgo en el seguro de daños (Santiago, Thomson-Reuters).

Ríos, Roberto (2015): “Artículo 542, Carácter imperativo de las normas, en Ríos Roberto (director), El Contrato de seguro, Comentarios al Título VIII Libro II del Código de Comercio (Santiago, Thomson Reuters).

Sánchez Calero, Fernando et al (2110): Artículo 10, Deber de declaración del riesgo, en Sánchez Calero (director) Ley de contrato de seguro, comentarios a la ley 50/1980 de 08 de octubre y sus modificaciones (Madrid, Thomson-Reuters).

Vivante, Cesare (1922): Del contratto di assicurazione (Torino, Unione Tipografico, Editrice-Torinese). 